\title{
A propos de la recherche sur la pédagogie de l'enseignement supérieur
}

Nicole Rege Colet et Christelle Lison

\section{(2) OpenEdition}

Édition électronique

URL : http://journals.openedition.org/ripes/894

DOI : 10.4000/ripes.894

ISSN : 2076-8427

Éditeur

Association internationale de pédagogie universitaire

Référence électronique

Nicole Rege Colet et Christelle Lison, «A propos de la recherche sur la pédagogie de l'enseignement supérieur », Revue internationale de pédagogie de l'enseignement supérieur [En ligne], 30(3) | 2014, mis en ligne le 01 décembre 2014, consulté le 25 septembre 2020. URL : http://journals.openedition.org/ ripes/894 ; DOI : https://doi.org/10.4000/ripes.894

Ce document a été généré automatiquement le 25 septembre 2020.

Article L.111-1 du Code de la propriété intellectuelle. 


\title{
A propos de la recherche sur la pédagogie de l'enseignement supérieur
}

\author{
Nicole Rege Colet et Christelle Lison
}

1 Les six articles de ce troisième numéro 2014 de RIPES illustrent différentes approches de la recherche en pédagogie de l'enseignement supérieur. La question de savoir ce qu'est la recherche en éducation nous occupent tous depuis fort longtemps. Les querelles d'écoles ne manquent pas. Il y a, d'un côté, ceux qui argumentent que seule la recherche expérimentale peut prétendre au statut de recherche, soit une recherche hypothético-déductive qui fonde sa connaissance probabiliste sur une empirie méthodologique robuste. Il y a, de l'autre côté, ceux qui défendent que la recherche sur les pratiques doit être polyforme et qu'il existe plusieurs manières de questionner les réalités complexes ainsi que les pratiques sociales et professionnelles qui les caractérisent.

2 Le développement pédagogique en enseignement supérieur est un champ de réflexions et de pratiques croisées. De fait, la recherche expérimentale ne peut pas, à elle seule, en rendre compte, ni fournir des outils pour en saisir les complexités et encore moins pour éclairer les pratiques sociales. Comme le souligne Cyrulnik (2014), la recherche expérimentale et le savoir fragmenté sont bien plus utiles pour faire une carrière académique ; la recherche expérimentale n'aide pas le praticien à intégrer les données, à interpréter la complexité et à dégager du sens. C'est pourquoi, au sein de la grande famille de la pédagogie de l'enseignement supérieur, nous sommes nombreux à plaider pour le SoTL (Scholarship of Teaching and Learning) comme approche de la recherche. Pour rappel, le SoTL désigne une démarche qui développe un discours savant sur les pratiques de l'enseignement et de l'apprentissage (Rege Colet \& Fanghanel, 2014).

Les six articles qui suivent témoignent, selon nous, de la diversité du SoTL et c'est avec plaisir et aussi fierté que nous les présentons à la communauté de la pédagogie de l'enseignement supérieur. Vous y trouverez différentes manières de récolter des 
données, de les analyser et de les interpréter pour accéder aux conceptions et aux approches qui mettent en mouvement les acteurs de l'enseignement supérieur.

Nous commencerons par une enquête auprès des diplômés d'une formation à l'enseignement conduite par Diane Leduc, Louise Ménard et Eric Le Coguiec de l'Université de Québec à Montréal. Au cours de leurs études, les diplômés ont pu expérimenter différentes approches de l'enseignement. Qu'en reste-t-il après la formation? L'enquête s'intéresse au transfert des outils et des modèles pédagogiques expérimentés pendant la formation vers les pratiques pédagogiques des diplômes devenus enseignants. Quel transfert des compétences? Et à quelles conditions?

Manon Jendly, Stéphanie Loup et Romain Voisard de l'Ecole des sciences criminelles de l'Université de Lausanne, Suisse, rendent compte d'une expérience pédagogique, le laboratoire de criminalité. Les étudiants qui fréquentent ce laboratoire se trouvent plongés dans les réalités du monde professionnel puisqu'ils se voient confiés un mandat réel qui sera au cœur de leurs apprentissages. L'intérêt de cet article réside aussi dans la démarche d'écriture; ces trois auteurs ont été accompagnés par trois conseillers pédagogiques de l'Université de Lausanne, Denis Berthiaume, Jean-Moïse Rochat et Emmanuel Sylvestre qui figurent à leurs côtés comme auteurs. Outre le compte rendu de pratique, l'article présente les outils développés pour comprendre comment les étudiants ont perçu cet enseignement: ici, l'évaluation de l'enseignement par les étudiants est centrée sur l'expérience formative des étudiants, un exemple de recherche évaluative.

6 Thierry Soubrié de l'Université de Grenoble, France, étudie comment le recours à Twitter peut soutenir la motivation des étudiants. L'analyse des usages autour de Twitter se fait à l'aune des théories motivationnelles afin d'étudier les liens entre interactions, engagement et persévérance aux études. L'analyse de contenus croise les apports d'un questionnaire pour accéder au vécu des étudiants.

7 Stéphane Balas du CNAM Paris et Priscilla Riem de l'Université Paris Descartes s'intéressent au déploiement de l'approche-programme dans les écoles d'ingénieurs et plus particulièrement au recours à des référentiels de compétences pour développer des parcours de formation. Ils examinent le travail de formalisation et d'explication qui s'opère lorsque les institutions s'engagent dans l'élaboration de parcours professionnels et dans le choix des approches pédagogiques cohérentes avec les intentions de formation. Ils montrent combien il peut être difficile de s'approprier les outils conceptuels d'une pédagogie centrée sur les apprentissages des étudiants et d'aligner de manière cohérente les outils.

Joëlle Demougeot-Lebel de l'Université de Bourgogne, France, nous propose de creuser l'idée de l'existence d'une génération $Y$. Sa recherche porte sur des enseignants que l'on pourrait qualifier d'enseignants de la génération Y. Elle cherche à comprendre si, au nom de cette appartenance, leurs pratiques pédagogiques sont innovantes et reflètent les attributs technologiques que l'on associe à la génération Y. Les résultats sont surprenants!

9 Et pour finir, la recherche de Léon Harvey, Marie-Hélène Hébert et Catherine Simard de l'Université du Québec à Rimouski nous ramène vers le monde de la recherche quantitative prouvant, par là, que la recherche appliquée en pédagogie de l'enseignement supérieur peut aussi accueillir des recherches quantitatives pour répondre aux questions que nous nous posons sur le développement de la qualité de l'enseignement et de l'apprentissage. Leur vaste étude, très technique, recourt à une 
analyse factorielle des dossiers des étudiants pour tester la validité et la fiabilité des évaluations des apprentissages des étudiants.

10 Strasbourg et Sherbrooke, le 17 novembre 2014

Nicole Rege Colet et Christelle Lison

\section{BIBLIOGRAPHIE}

Cyrulnik, B. (2014). Les âmes blessées. Paris : Odile Jacob.

Rege Colet, N. \& Fanghanel, J. (2014). Faire de la recherche appliquée sur ses enseignements. Dans N. Rege Colet \& D. Berthiaume (Eds.), La pédagogie de l'enseignement supérieur ; repères théoriques et applications pratiques. Tome 2. Se développer au titre d'enseignant du supérieur (pp. 221-234). Berne : Peter Lang

\section{AUTEURS}

NICOLE REGE COLET

Strasbourg

\section{CHRISTELLE LISON}

Sherbrooke 